\title{
Science Opportunities offered by Mercury's Ice-Bearing Polar Deposits
}

\author{
Ariel N. Deutsch \\ Brown University \\ Ariel_Deutsch@brown.edu \\ (401) 863-1000
}

\author{
Nancy L. Chabot \\ JHU Applied Physics Laboratory \\ Nancy.Chabot@jhuapl.edu \\ (240) 228-5558
}

\section{with support from}

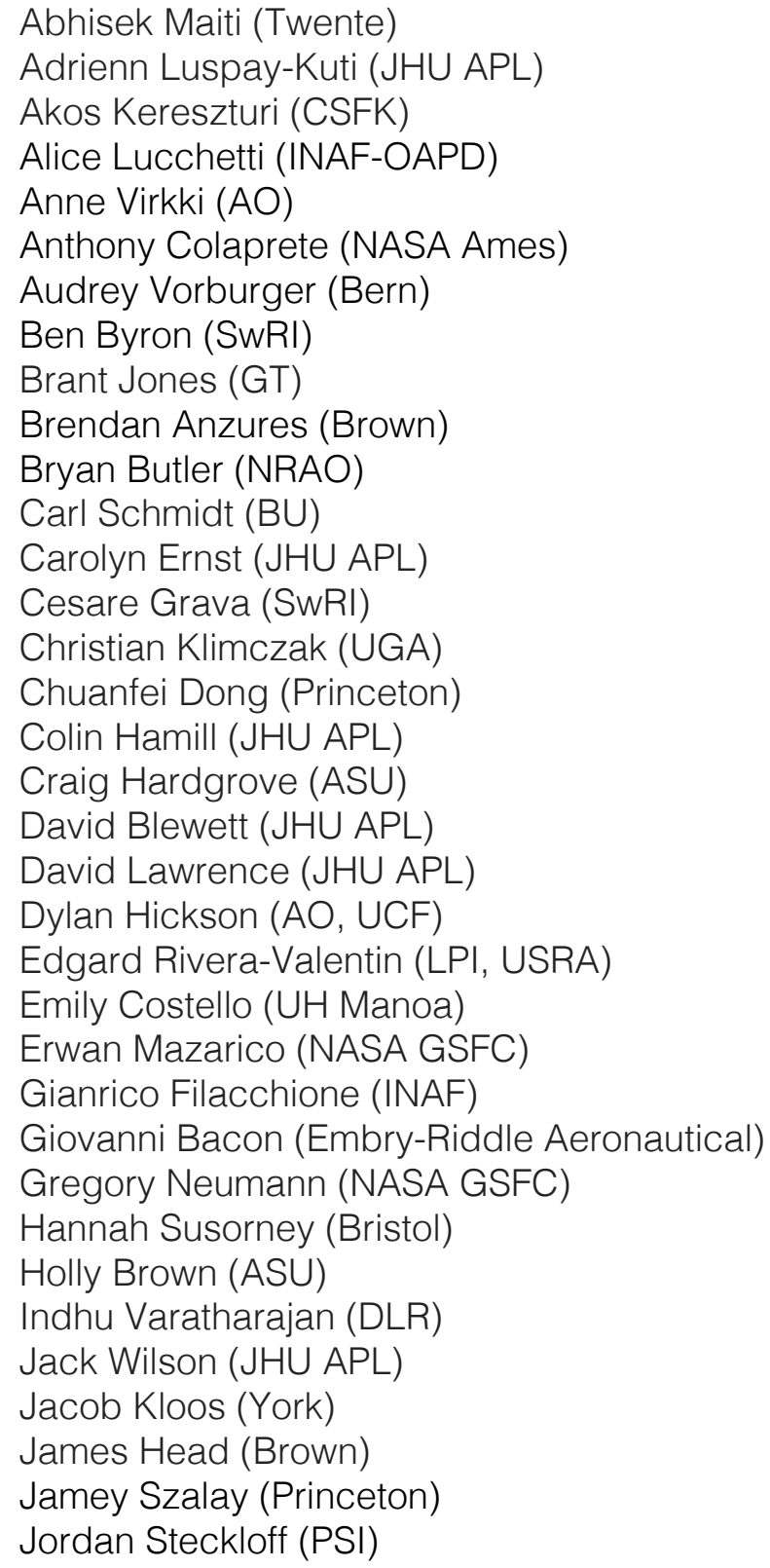

Kelly Miller (SwRI)

Kevin Cannon (UCF)

Kristen Luchsinger (NMSU)

Lior Rubanenko (Stanford)

Lizeth Magana (UTSA, SwRI)

Margaret Landis (CU Boulder)

Maria Gritsevich (FGl)

Mark Schneegurt (WSU)

Martin Slade (JPL/Caltech)

Matthew Siegler (PSI)

Maurizio Pajola (INAF-OAPD)

Menelaos Sarantos (NASA GSFC)

Michael Poston (SwRI)

Michael Sori (Purdue)

Mona Delitsky (CA Specialty Engineering)

Noemi Pinilla-Alonso (FSI, AO, UCF)

Norbert Schorghofer (PSI)

Parvathy Prem (JHU APL)

Paul Byrne (NC State)

Paul Hayne (CU Boulder)

Paul Lucey (UH Manoa)

Peter James (Baylor)

Petr Pokorný (CUA, NASA GSFC)

Ronald Vervak, Jr. (JHU APL) Rosemary Killen (NASA GSFC)

Ross Potter (Brown)

Sean Solomon (Columbia)

Shashwat Shukla (Twente)

Sriram Bhiravarasu (ISRO)

Steven Hauck (CWRU)

Thomas Orlando (GT)

Timothy Stubbs (NASA GSFC)

Valentin Bickel (ETH Zurich, MPS Goettingen)

Vincent Eke (Durham)

William Farrell (NASA GSFC) 


\section{Executive summary}

The presence of meters-thick polar deposits exposed directly on the surface of Mercury provides unique science opportunities that should be prioritized in the next decade of planetary exploration. The poles of Mercury provide a natural laboratory for understanding the chemical, physical, and thermal processes that have governed the supply, retention, modification, and loss of water and other volatiles delivered to the inner solar system through time. Polar deposits on Mercury are composed primarily of water ice, and the additional and coincident presence of organic-rich frozen volatiles exposed on the surface offers a nearby opportunity to study the chemistry of extremely lowtemperature, icy environments applicable to the Moon, asteroids, comets, and icy worlds of the outer solar system. Understanding the processes responsible for the delivery and storage of volatiles on Mercury would greatly enhance our ability to understand the origin and distribution of resources across the inner solar system, including at the lunar poles.

\section{Introduction}

Earth-based radar observations of high-backscatter materials provided the first evidence of water ice present at Mercury's polar regions (e.g., Slade et al., 1992; Harmon and Slade, 1992; Butler et al., 1993; Harmon et al., 2011). In the last decade, the MErcury Surface, Space ENvironment, GEochemistry, and Ranging (MESSENGER) spacecraft revealed that these radar-bright materials are cold-trapped within permanently shadowed regions (PSRs, e.g., Deutsch et al., 2016; Chabot at al., 2018), where temperatures calculated from measured topography and insolation models are sufficiently cold to sustain water ice (Paige et al., 2013). The detected enhanced levels of hydrogen are consistent with water ice as the primary constituent of the deposits (Lawrence et al., 2013). The distinct reflectance properties of some of Mercury's polar deposits indicate that they are exposed directly at the surface (e.g., Neumann et al., 2013; Chabot et al., 2016; Deutsch et al., 2017), providing favorable observation opportunities and direct access to thick and relatively pristine material (e.g., Butler et al., 1993; Harmon, 2007; Black et al., 2010; Lawrence et al., 2013; Rubanenko et al., 2019; Susorney et al., 2019), whereas at other deposits, the water ice is covered by frozen organic-rich volatiles (Zhang and Paige, 2009; Delitsky et al., 2017) (Fig. 1).

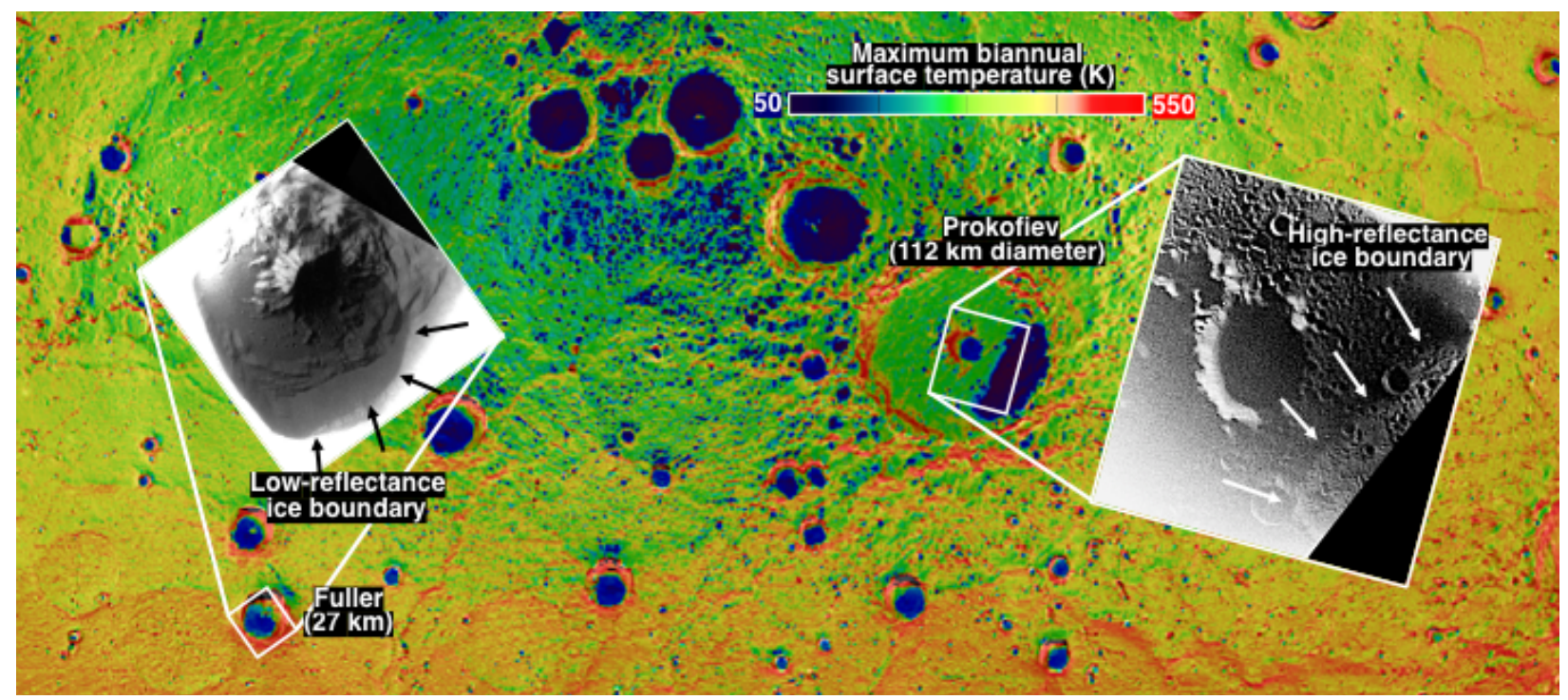

Fig. 1. Mercury's north polar deposits feature large expanses of exposed water ice (e.g., Prokofiev crater) as well as other low-reflectance volatiles (e.g., Fuller crater). 
Excitingly, the abundant polar deposits likely preserve some of the most recently delivered and sequestered volatiles in the inner solar system (Lawrence et al., 2013; Deutsch et al., 2019). Therefore, chemical measurements of these deposits would provide important insight into the nature of their materials, potentially before the deposits have been substantially chemically, thermally, or physically altered. Overall, the polar environments on Mercury provide scientifically compelling opportunities for studying the record of cold-trapped volatiles, as well as the ongoing micrometeoroid bombardment and solar wind-regolith interactions that are affecting the evolution of Mercury's surface volatiles and exosphere, serving as an accessible environment to investigate these processes that operate across the solar system.

One of the major breakthroughs in Mercury science over the past decade has been the multiple lines of evidence that Mercury's polar regions host water ice and other volatiles. Understanding the volatile budget of Mercury was recognized as an important open question in the previous Decadal Survey and remains an important priority. Here we discuss the unique science opportunities that motivate the further study of Mercury's polar deposits. Addressing the top-level science questions outlined below has major implications for understanding the nature of volatiles on Mercury, as well as the sources, evolution, and inventory of volatiles in the solar system through time.

\section{Relevance to community objectives}

Understanding the nature and extent of the polar deposits on Mercury has important and wide-reaching implications for planetary science. The nature of cold-trapped ices can provide insight into the origin and inventory of lunar volatiles, and even the delivery of water and organics to early Earth and Mars, and therefore is relevant for lunar, Earth, and other terrestrial planetary objectives. Mercury's youngest polar deposits are likely derived from an impact or impact events (Ernst et al., 2018), and therefore provide an opportunity to study outer solar system volatiles and organics. The polar deposits also provide an opportunity to study the very cold chemistry occurring between volatiles, organics, minerals, and high-energy particles, which is of importance to questions in astrobiology and the formation and evolution of outer solar system objects.

\section{Top-level science questions}

\section{What are the origin and age of polar deposits on Mercury, and the relative contributions of impacts, solar wind, and outgassing?}

Polar deposits on Mercury appear to be remarkably pure, given that Earth-based telescopic observations indicate the radar-bright ice deposits have impurities of $<5 \%$ by volume (Butler et al., 1993). Such a high degree of purity suggests that the radar-bright polar deposits were emplaced relatively recently, within the last 50-200 Myr, because they have not been substantially modified by regolith gardening (Crider and Killen, 2005; Costello et al., 2020). Investigating the chemical composition of Mercury's polar ices is essential for determining the origin of this pure ice, given that the speciation and isotopic ratios of cold-trapped volatiles will vary depending on their origin. These properties could be directly compared with the chemical composition of terrestrial and lunar volatiles, as well as cometary materials, asteroids and meteorites, Kuiper belt objects, and ices on Ceres and outer solar system satellites, thereby providing insight into the origin of inner solar system volatiles.

While the most poleward deposits on Mercury have high-reflectance properties suggesting that water ice is directly exposed at the surface (Neumann et al., 2013; Deutsch et al., 2017), the majority of ice deposits are insulated by a 10-30 cm-thick 
layer of low-reflectance volatiles (Lawrence et al., 2013) (Fig. 2). The reflectance properties (Chabot et al., 2016) and thermal environments (Zhang and Paige, 2009; Paige et al., 2013) of these materials suggest that they are composed of frozen volatiles other than water, potentially including simple organic compounds as well as macromolecular carbonaceous materials. These low-reflectance volatiles may have been delivered simultaneously with the water deposits in one or more impact events (e.g., Zhang and Paige, 2009), or processed in situ under the harsh radiation environment on Mercury (e.g., Delitsky et al., 2017). Thus, the chemistry of both the seemingly pure water-ice deposits and of the intriguing low-reflectance ices will help resolve the origin of Mercury's polar deposits.

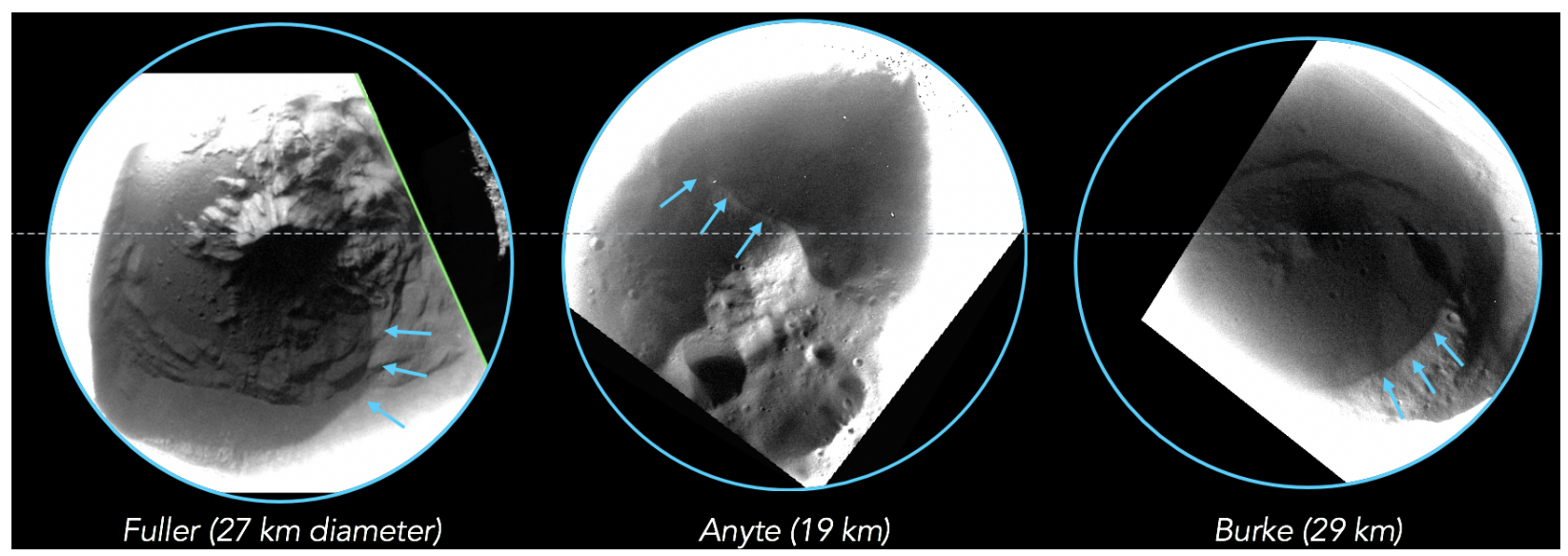

Fig. 2. The majority of water-ice deposits on Mercury are insulated by a thin layer of lowreflectance, likely organic-rich materials. All polar deposits appear to have very sharp reflectance boundaries, designated by the arrows.

Both exogenic and endogenic processes may be responsible for delivering volatiles to Mercury's polar regions. If the polar deposits were delivered by a single recent $(<100$ Ma) large impact event (e.g., Ernst et al., 2018), then the polar deposits may provide access to outer solar system materials at a relatively close distance. If water is also being supplied through the thermal transformation of mineral-bound hydroxyl groups by implanted solar-wind protons (e.g., Starukhina, 2006; Jones et al., 2020), then the polar deposits also provide direct access to studying the modern-day volatile cycle operating on Mercury, as well as on the Moon, Galilean satellites, and other airless bodies.

Finally, studying the detailed volatile speciation and concentration in stratigraphy can also help provide important insight into the timing and temporary duration of atmospheric development from impacts and potentially volcanic sources. This information is particularly important when considering how transient atmospheric generation relates to volatile transport and delivery, radiation shielding, and the evolution of habitable conditions on early terrestrial planets.

A major open question regarding the volatile inventories on Mercury (and elsewhere) is whether the polar cold traps are in a state of relative equilibrium, net accumulation, or net loss. Detailed studies of the stratigraphy of Mercury's meters-thick polar deposits can provide important insight into the relative strengths of the processes governing the flux state of the polar volatiles. Overall, studying the chemical and physical properties of the polar deposits will provide an understanding of how the planet's volatile inventory has developed through time, perhaps most recently through the quasi-continuous nature of micrometeoroid and solar-wind bombardment (Pokorný et al., 2018; Jones et al., 2020), a single large, water-rich impact (Ernst et al., 2018), or both. Such investigations 
will allow us to better understand and track Mercury's volatile inventory back through time, during periods of higher impact fluxes and enhanced solar wind intensities (e.g., Nesvorný et al., 2017; Pognan et al., 2018), during volcanic outgassing events (e.g., Head et al., 2011), and during the possible development and evolution of a global magma ocean (e.g., Vander Kaaden and McCubbin, 2015).

\section{What are the processes modulating the retention, modification, and loss of cold volatile-rich surfaces?}

The polar environment at Mercury provides a natural laboratory for studying processes actively modulating the retention, modification, and loss of volatiles, and these processes have direct implications for understanding the volatile cycles on other airless bodies, including the Moon and Ceres. The relatively higher thermal gradients, higher micrometeoroid flux, higher solar irradiation, and variable magnetic environment make Mercury a particularly interesting place to study surface modification processes (e.g., Domingue et al., 2015; Jones et al., 2020).

A primary factor governing the retention of polar volatiles is temperature (e.g., Vasavada et al., 1999; Paige et al., 2013; Rubanenko et al., 2018), which modulates the rates of volatile diffusion and sublimation (e.g., Hibbitts et al., 2011; Schorghofer and Aharonson, 2014). Additionally, the relatively high rate of micrometeoroid impact bombardment at Mercury is predicted to affect the purity and distribution of the ice deposits, as well as the preservation of thermal lag deposits (Crider and Killen, 2005; Costello et al., 2020). Therefore, characterizing polar thermal environments, space weathering rates, and the distribution of polar volatiles will provide key information on the processes that affect the retention of volatiles on Mercury and other airless bodies.

The chemical and physical nature (i.e., albedo properties, surface texture) of the ice also provide an important record of how it has been modified by the solar and space weathering environment through bombardment, irradiation, and thermal stresses (e.g., Domingue et al., 2015; Delitsky et al., 2017; Farrell et al., 2019; Costello et al., 2020). The occurrence, abundance, and ratio of volatiles and intermixed regolith provide information on surface modifications, including dust migration. The various space weathering processes operating at Mercury affect the durability of volatiles as a sustainable resource for future exploration, as they drive modifications in volatile distribution and abundance through time, and thus these topics are also of particular interest to lunar science and exploration.

\section{What chemical reactions are occurring between organic compounds, water ice, and other frozen volatiles at very low temperatures?}

Perhaps one of the most intriguing and unique opportunities offered by Mercury's polar deposits is the ability to study the chemical reactions occurring between water, other volatiles (e.g., S, Hg), and organic compounds at extremely cold polar temperatures, which can reach $50 \mathrm{~K}$ or lower in PSRs (Fig. 1; Zhang and Paige, 2009; Paige et al., 2013). The presence of $\mathrm{H}_{2} \mathrm{O}$ affects low-temperature reactivity, which may control the formation of complex molecules and the variety of radiolytically-formed products in the polar deposits. Thermally-driven solid-phase chemistry at low temperatures can form products that are different than those synthesized by radiolysis; therefore, characterizing how radiolytically- and thermally-driven chemistry occurs in the polar deposits would be crucial to understand their chemical evolution and preservation through time. The study of irradiated volatile deposits in a natural context would also provide important ground truth for experimentally-based hypotheses on the origin of organic material in primitive bodies, as laboratory irradiation of ice-organic mixtures at low temperatures and 
pressures have demonstrated the formation of prebiotic molecules similar to those identified in the soluble or volatile fractions of meteorites and comets, including amino acids (Bernstein et al., 2002; Muñoz Caro et al., 2002), fatty acids (Dworkin et al., 2001), and nucleobases (Nuevo et al., 2009). Thus, Mercury's polar deposits may provide a nearby analog for cold, primitive bodies and outer solar system materials. For example, they may offer the opportunity to identify tholins, which are predicted to be abundant in the outer solar system from irradiated organics (e.g., Pinilla-Alonso et al., 2011; Cartwright et al., 2015), as well as organic-rich ice grains, which have been identified in Enceladus plume materials (Postberg et al. 2018) and Saturn's rings (Miller et al., 2020).

Additionally, studying the rates of chemical reactions would provide important new insight into the rates of formation and erosion of Mercury's low-reflectance volatiles. Ionizing radiation is well known to induce chemical and physical changes of adsorbed ice. Despite the weak magnetic field surrounding Mercury, ionizing radiation can still reach PSRs through photoelectron emission and grain charging (Farrell et al. 2019) and penetrate Mercury's surface (Benna et al. 2010). Interactions between high-energy particles and condensed volatiles have repeatedly been demonstrated to chemically modify pristine ices via chemistry involving non-thermal reactants such as hydrogen, oxygen, nitrogen, and carbon radicals (e.g., Herbst \& van-Dishoeck 2009; Kaiser et al., 2014). On Mercury, these interactions are expected to result in the synthesis of lowreflectance, complex organic molecules even at cryogenic temperatures.

The polar deposits offer an interesting opportunity to understand extreme conditions, with the coincident presence of volatiles and organics in low-temperature, high-radiation environments at a laboratory that is close to Earth. Understanding the formation of complex molecules on Mercury can help untangle the conditions that played a significant role in the evolution of our solar system.

\section{What are the physical and geomechanical properties of cold polar regolith and volatiles?}

Mercury's poles also provide a testing ground for resolving the physical and geomechanical properties of cold polar regolith and volatiles. Specifically, the ice phase, the grain size distribution, binding energies, and porosity of the regolith, and the density, volume, and stratigraphy of the ice deposits are not constrained, but these variables have important implications for understanding the planet's total volatile budget, and for interpreting ground-based and orbital observations of ice on Mercury, as well as on other airless bodies. For example, the presence of organics may have important alteration effects on the thermal properties of the ice deposits, which may be key in understanding differences between lunar and cerean volatiles, which seem to have less carbon contamination. Additionally, the phase of the ice (amorphous, crystalline, etc.) is poorly constrained, but is indicative of primordial material or material that has been modified by thermal or radiation processes. The potential presence of clathrates could help to retain species with higher volatility (e.g., $\left.\mathrm{CH}_{4}, \mathrm{CO}\right)$ that could otherwise sublimate away.

Additionally, analyzing the regolith porosity and binding energies will improve our understanding of the electrostatic and thermophysical environment of Mercury's coldtrapping regions (e.g., Domingue et al., 2015), helping us to quantify the regions available to the cold-trapping of volatiles, in micro-cold traps, in pore spaces, and along grain boundaries. Knowledge of water binding energies on Mercury's regolith is crucial to understanding the transport of water on the surface. The quantification of volatile binding energies and total binding sites will allow for the prediction of where and how much water can accumulate, such as in permanently shadowed and polar regions. Ultimately, understanding the regolith porosity and binding energies allows for the 
understanding of what governed the accretion of water and how space weathering affects potential binding sites.

\section{Conclusions and recommendations}

Our understanding of polar deposits on Mercury has drastically improved over the last decade as a direct result of the MESSENGER mission. This improvement has relied heavily on orbital observations of the north polar region due to MESSENGER's periapsis, but the south polar deposits are key to understanding the global inventory of polar volatiles (e.g., Chabot et al., 2018). Our understanding will continue to improve as the BepiColombo spacecraft enter orbit around Mercury in late 2025 and acquire higher resolution images and altimetry of the south polar region and thermal IR data. However, even with the measurements to be made by BepiColombo, the important scientific questions outlined here will still remain, and the additional, dedicated exploration of Mercury's polar deposits offer unique opportunities to address them. Therefore, we make the following recommendations to support these opportunities:

- Support research and analysis of Mercury's polar deposits, including laboratory and computational studies related to cold-trapped volatiles and MESSENGER data.

- Support the continued investigation of Mercury with the ESA/JAXA BepiColombo mission, taking any opportunities that arise to maximize the science achieved by the mission, such as by future Participating Scientist opportunities for the orbital phase of the mission. This is especially important because there are no other planned missions to Mercury currently.

- Expand Earth-based radar campaigns (e.g., with the high sensitivity S-band radar at Arecibo Observatory) to support the ground observations that can help refine our understanding of the composition, purity, porosity, and the horizontal and vertical distribution (and spatial variability) of polar ices, as well as possible changes in these properties over the last several decades.

- Develop the concept for and fly one or more missions to explore Mercury's polar deposits with landed assets to directly probe their composition, stratigraphy, and geophysical properties, refine structure and thermal measurements, and groundtruth orbital and Earth-based observations.

- In the longer term, develop cryogenic sample analysis, storage, and return capabilities for studying mercurian volatiles as well as analog materials, including volatiles from the lunar poles, icy satellites, comets, and even interstellar objects.

A fuller understanding of Mercury's polar deposits will provide critical insight into the origin, evolution, and inventory of solar system volatiles. After the knowledge gained by MESSENGER in the last decade, this coming decade is the time to take further advantage of this unique natural laboratory known to have large expanses of water ice and other volatiles accessible on the surface, waiting to be explored.

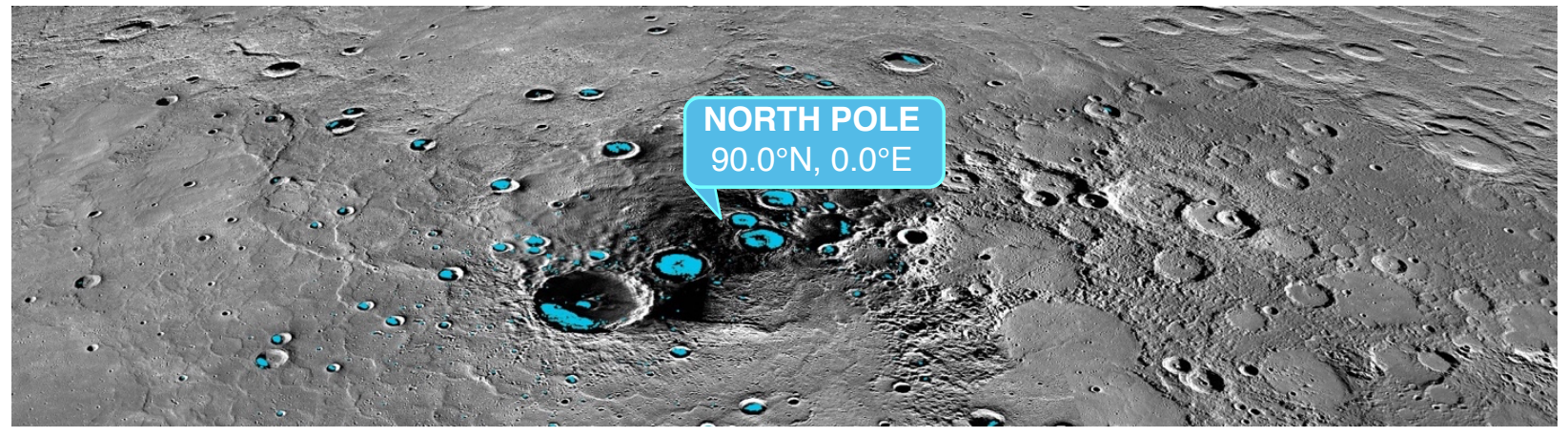




\section{References}

Benna, M., et al. (2010) /carus 209, 3-10. doi:10.1016/j.icarus.2009.11.036

Bernstein, M.P., et al. (2002) Nature 416, 401-403. doi:10.1038/416401a

Black, G.J., et al. (2010) Icarus 209, 224229. doi:10.1016/j.icarus.2009.10.009

Butler, B.J. (1997) JGRP 102, 1928319291. doi:10.1029/97JE01347

Cartwright, R.J., et al. (2015) /carus 257, 428-456.

doi:10.1016/j.icarus.2015.05.020

Chabot, N.L., et al. (2014) Geology 42, 1051-1054. doi:10.1130/G35916.1

Chabot, N.L., et al. (2016) GRL 43, 94619468. doi:10.1002/2016GL070403

Chabot, N.L., et al. (2018) JGRP 123, 666681. doi:10.1002/2017JE005500

Costello, E.S., et al. (2020) JGRP 125, e2019JE006172.

doi:10.1029/2019JE006172

Crider, D., \& Killen, R.M. (2005) GRL 32, L12201. doi:10.1029/2005GL022689

Delitsky, M.L., et al. (2017) /carus 281, 19 31. doi:10.1016/j.icarus.2016.08.006

Deutsch, A.N., et al. (2016) Icarus 280, 158-171. doi:10.1016/j.icarus.2016.06.015

Deutsch, A.N., et al. (2017) GRL 44, 92339241. doi:10.1002/2017GL074723

Deutsch, A.N., et al. (2019) EPSL 520, 2633. doi:10.1016/j.epsl.2019.05.027

Domingue, D.L., et al. (2014) SSR 181, 121 -214. doi:10.1007/s11214-014-0039-5

Dworkin, J.P., et al. (2001) PNAS 98, 815819. doi:10.1073/pnas.98.3.815

Ernst, C.M., et al. (2018) JGRP 123, 26282646. doi:10.1029/2018JE005552

Farrell, W.M., et al. (2019) GRL 46, 86808688. doi:10.1029/2019GL083158

Harmon, J.K. (2007) SSR 132, 307-349. doi:10.1007/s11214-007-9234-y

Harmon, J.K., \& Slade, M.A. (1992) Science 258, 640-643. doi:10.1126/science.258.5082.640

Harmon, J.K., et al. (2011) Icarus 211, 3750. doi:10.1016/j.icarus.2010.08.007

Head, J.W., et al. (2011) Science 333, 1853-1856. doi:10.1126/science. 1211997

Herbst, E., \& van Dishoeck, E.F. (2009) Ann Rev AA 47, 427-480. doi:10.1146/annurev-astro-082708101654

Hibbitts, C.A., et al. (2011) Icarus 213, 6472. doi:10.1016/j.icarus.2011.02.015 Jones, B.M., et al. (2020) ApJ Lett 891, L43. doi:10.3847/2041-8213/ab6bda

Kaiser, R.I., et al. (2014) PCCP 16, 33993424. doi:10.1039/C3CP54255F

Lawrence, D.J., et al. (2013) Science 339, 292-296. doi:10.1126/science.1229953

Miller, K.E., et al. (2020) Icarus 339, 113595.

doi:10.1016/j.icarus.2019.113595

Muñoz Caro, G.M., et al. (2002) Nature 416, 403-406. doi:10.1038/416403a

Nesvorný, D., et al. (2017) ApJ 153, 103. doi:10.3847/1538-3881/153/3/103

Neumann, G.A., et al. (2013) Science 339, 296-300. doi:10.1126/science.1229764

Nuevo, M., et al. (2009) Astrobio 9, 683695. doi:10.1089/ast.2008.0324

Paige, D.A., et al. (2013) Science 339, 300-303. doi:10.1126/science.1231106

Pinilla-Alonso, N., et al. (2011) Icarus 215, 75-82. doi:10.1016/j.icarus.2011.07.004

Pognan, Q., et al. (2018) ApJ 856, 53. doi:10.3847/1538-4357/aaaebb

Pokorný, P., et al. (2018) ApJ 863, 31. doi:10.3847/1538-4357/aad051

Postberg, F., et al. (2018) Nature 558, 564 -568. doi:10.1038/s41586-018-0246-4

Rubanenko, L., et al. (2018) JGRP 123, 2178-2191. doi:10.1029/2018JE005644

Rubanenko, L., et al. (2019) Nat Geo 12, 597. doi:10.1038/s41561-019-0405-8

Schorghofer, N., \& Aharonson, O. (2014) ApJ 788, 169. doi:10.1088/0004637X/788/2/169

Slade, M.A., et al. (1992) Science 258, 635-640. doi:10.1126/science.258.5082.635

Starukhina, L.V. (2006) ASR 37, 50-58. doi:10.1016/j.asr.2005.04.033

Susorney, H.C.M., et al. (2019) Icarus 323, 40-45. doi:10.1016/j.icarus.2019.01.016

Vander Kaaden, K.E., \& McCubbin, F.M. (2015) JGRP 120, 195-209. doi:10.1002/2014JE004733

Vasavada, A.R., et al. (1999) /carus 141, 179-193. doi:10.1006/icar.1999.6175

Zhang, J.A., \& Paige, D.A. (2009) GRL 36, L16203. doi:10.1029/2009 